\title{
Frontotemporal dementia, right temporal atrophy variant
}

INSERM

\section{Source}

INSERM. (1999). Orphanet: an online rare disease and orphan drug data base.

Frontotemporal dementia, right temporal atrophy variant. ORPHA:293848

Right temporal lobar atrophy (RT LA) is an anatomic variant of frontotemporal dementia (FTD), characterized by behavioral dysfunction, personality changes, episodic memory loss, and prosopagnosia; attributable to an asymmetrical predominantly right-sided, frontotemporal atrophy. 